\title{
EXTREME POINTS OF A CLASS OF SUBORDINATE FUNCTIONS
}

\author{
T. SHEIL - SMALL
}

\begin{abstract}
It is shown that, if $F(z)$ is subordinate to $H((1+z) /(1-z))$ in the unit disc, where $H(w)$ is a quadratic polynomial for which $H^{\prime}(w) \neq 0$ in the right
\end{abstract} half-plane, then

$$
F(z)=\int_{T} H\left(\frac{1+z e^{-i t}}{1-z e^{-i t}}\right) d \mu
$$

for a suitable probability measure $\mu$ on the unit circle $T$.

In [1, Problem 5.59], the problem was raised of describing functions $G(z)$ analytic in $U=\{|z|<1\}$ with the property that the extreme points of the closed convex hull of the class

$$
S_{G}=\{F: F \prec G\}
$$

had the form $G\left(z e^{i t}\right)(0 \leqslant t<2 \pi)$. Here $F \prec G$ means $F$ subordinate to $G$. It is easily seen that the functions $G\left(z e^{i t}\right)$ are extreme points. The general problem of describing support points and extreme points of subordination families has recently been taken up by Hallenbeck and MacGregor [2]. In this note we will establish a result, which we claimed without proof in [1]. The method of proof is one which may generalise to other classes.

THEOREM. Let $G(z)$ be a function of the form $H((1+z) /(1-z))$, where $H(w)$ is a univalent, quadratic polynomial in $\{\operatorname{Re} w>0\}$. Then the extreme points of the closed convex hull of $S_{G}$ have the form $G\left(z e^{i t}\right)(0 \leqslant t<2 \pi)$.

Proof. Let $H(w)=A_{0}+A_{1} w+A_{2} w^{2}$. The condition for univalence in $\{\operatorname{Re} w>$ $0\}$ is

$$
\operatorname{Re}\left(A_{1} / A_{2}\right) \geqslant 0 \text {. }
$$

Thus

$$
\begin{aligned}
G(z) & =A_{0}-A_{1}+A_{2}+2 A_{1} \frac{1}{1-z}+4 A_{2} \frac{z}{(1-z)^{2}} \\
& =A_{0}+A_{1}+A_{2}+\sum_{n=1}^{\infty} 2\left(A_{1}+2 n A_{2}\right) z^{n} .
\end{aligned}
$$

Received by the editors June 7, 1983.

1980 Mathematics Subject Classification. Primary 30C80; Secondary 30E20.

(c)1984 American Mathematical Society $0002-9939 / 84 \$ 1.00+\$ .25$ per page 
Without loss of generality we may assume $A_{0}+A_{1}+A_{2} \neq 0$. Then

$$
\left|A_{1}+2 n A_{2}\right| \geqslant 2\left|A_{2}\right|
$$

and so the convolution inverse

$$
G_{i}(z)=\frac{1}{A_{0}+A_{1}+A_{2}}+\sum_{n=1}^{\infty} \frac{1}{2\left(A_{1}+2 n A_{2}\right)} z^{n}
$$

is analytic in $U$. For $F \in S_{G}$ we show that

$$
\operatorname{Re}\left(G_{i} * F\right)(z)>1 / 2 \quad(z \in U) .
$$

Writing $P=G_{i} * F$ we have $P(0)=1$, so (6) implies

$$
F(z)=(G * P)(z)=\frac{1}{2 \pi} \int_{0}^{2 \pi} G\left(z e^{i t}\right) d \mu(t),
$$

where $\mu(t)$ is a nondecreasing function on $[0,2 \pi]$ with $\mu(2 \pi)=\mu(0)+2 \pi$. Here we have applied the Herglotz representation for $P$. The theorem clearly follows from (7). Now if (6) does not hold, we deduce from the Clunie-Jack lemma [3] that $\exists \xi \in U, c$ real with

$$
P(\xi)=\frac{1}{2}+i c, \quad \xi P^{\prime}(\xi)=-d \leqslant-\left(\frac{1}{4}+c^{2}\right) .
$$

Then

$$
\begin{aligned}
F(\xi) & =(G * P)(\xi)=A_{0}-A_{1}+A_{2}+2 A_{1} P(\xi)+4 A_{2} \xi P^{\prime}(\xi) \\
& =A_{0}+A_{1}(2 i c)+A_{2}\left(-4 c^{2}\right)-4 \alpha A_{2}=H(2 i c)-4 \alpha A_{2},
\end{aligned}
$$

where $\alpha \geqslant 0$. But

$$
F(\xi)=H(w),
$$

where $\operatorname{Re} w>0$. Hence the nonpositive real number

$$
\begin{aligned}
-4 \alpha & =\frac{F(\xi)-H(2 i c)}{A_{2}}=\frac{A_{1}}{A_{2}}(w-2 i c)+w^{2}-(2 i c)^{2} \\
& =(w-2 i c)\left(\frac{A_{1}}{A_{2}}+w+2 i c\right)
\end{aligned}
$$

is the product of two numbers of positive real part, which is impossible. Therefore (6) holds and the proof is complete.

\section{REFERENCES}

1. D. A. Brannan and J. G. Clunie, eds., Aspects of contemporary complex analysis, Academic Press, New York, 1980.

2. D. J. Hallenbeck and T. H. MacGregor, Support points of families of analytic functions described by subordination, Trans. Amer. Math. Soc. 278 (1983), 523-546.

3. I. S. Jack, Functions starlike and convex of order $\alpha$, J. London Math. Soc. 3 (1971), 469-474.

Department of Mathematics, University of York, Heslington, York, Y01 5DD, ENGLAND 